\title{
DER UMWELTSCHUTZ AUF HOHER SEE - INTERNATIONALE WIE NATIONALE MASSNAHMEN UND BESTREBUNGEN
}

\author{
Von RÜDIGER WOLFRUM
}

Die 3. Seerechtskonferenz der Vereinten Nationen hat ihre Beratungen am 17. März 1975 in Genf fortgesetzt. Das Interesse aller konzentriert sich dabei wohl auf die Frage, zu welchen Ergebnissen sie hinsichtlich des Fischfangs und des Meeresbergbaus gelangen wird. Demgegenüber ist die Anteilnahme an der zukünftigen Regelung des maritimen Umweltschutzes ein wenig in den Hintergrund getreten, obwohl von daher tiefgreifende Einschränkungen vor allem der Schiffahrt, aber auch des Meeresbergbaus zu befürchten sind.

Die Fragen des maritimen Umweltschutzes, die mit in den Zuständigkeitsbereich des 3. Ausschusses der Konferenz fallen, sind vor allem wegen der verschiedenartigen Ursachen der Meeresverschmutzung außerordentlich komplex. Die Meeresverschmutzung läßt sich in Anlehnung an die Beratungen in der 3. Seerechtskonferenz auf sechs Hauptquellen zurückführen ${ }^{1}$; es ist die Wasserverunreinigung von Land aus, durch die Luft, durch Abfallablagerungen (dumping), die Verschmutzung von Schiffen, sei es als Folge eines Unfalls (Ölaustritt bei Tankerunfällen) oder als Begleiterscheinung der Schiffsroutine (Reinigung der Bilgen und der Öltanks), bei der Ausbeutung des Meeresbodens und von Anlagen, die nicht dem Meeresbergbau dienen, zu unterscheiden.

Die Schwierigkeiten der Seerechtskonferenz bei einer Regelung dieses Komplexes bestehen darin, daß sie trotz der so verschiedenen Ursachen ein umfassendes Übereinkommen erarbeiten muß, soll ein wirklicher Schutz der maritimen Umwelt gewährleistet sein ${ }^{2}$. Hinzu kommt, daß die Bestrebungen der interessierten Staaten, ähnlich wie bei der Aufteilung der Fischereirechte und des Meeresbergbaus außerordentlich kontrovers sind. Im wesentlichen lassen sich die Meinungsverschiedenheiten auf die allgemeine Frage reduzieren, in welchem Umfang die Rechte der Küstenstaaten verstärkt und ihnen Befugnisse auch für den Bereich der Hohen See zugebilligt werden sollen. Einige Staaten, wenn auch in geringerem Umfang, als dies bei der Ausdehnung der Fischereigrenzen zu beobachten ist, haben sogar, ohne eine internationale Lösung dieses Problems abzuwarten, einseitig Maßnahmen ergriffen, die auch Teile der Hohen See einbeziehen und damit ihrer Kontrolle unterwerfen. Dieses Vorgehen wurde im wesentlichen damit begründet, daß die existierenden internationalen Übereinkommen ebenso wie die von der IMCO vorgelegten, aber noch nicht in Kraft getretenen Konventionsentwürfe gegen die Meeresverschmutzung von Schiffen, wegen einer zu starken Betonung des Flaggenstaatsprinzips nicht in der Lage seien, einen genügenden Schutz der Meere und Küsten zu gewährleisten ${ }^{3}$.

1 Doc. A/Conf. 62/C.3/L. 15.

2 Vgl. dazu General Guidelines and Principles for the Preservation of the Marine Environment der Inter-Governmental Working Group on Marine Pollution, 2nd Sess. 1971 Ottawa, in: Barros and Johnston, The International Law of Pollution, New York, London 1974, S. 323.

3 Vgl. Rede des Kanadischen Außenministers vor dem Unterhaus zur Einbringung des Arctic Waters Pollution Prevention Act, April 1970, Text in: Walter Pollution as a World Problem, Report of a Conference held at the University College of Wales, Aberystwyth, 11./12. Juli 1970, Appendix II S. 207; Preseinterview von Premierminister Trudeau zu dem gleichen Thema vom 8. April 1970, in: ILM, Vol. 9 (1970), S. 600 . 
1. Die bisherige Bekämpfung der Meeresverschmutzung auf internationaler Ebene Bereits die Genfer Übereinkommen über die Hohe See (Art. 24), über den Festlandsockel (Art. 5, Abs. 7) sowie über die Fischerei und die Erhaltung der lebenden Schätze der Hohen See (Art. 1, Abs. 2) enthalten Bestimmungen zum Schutze der maritimen Umwelt. Sie machen es den Staaten allerdings nur ganz allgemein zur Pflicht, hinsichtlich der Schiffahrt und der Ausbeutung des Meeresgrundes wie des Festlandsockels Vorkehrungen zur Erhaltung der maritimen Umwelt zu treffen. Dagegen wurde das Problem der Abfallbeseitigung lediglich für radioaktive Stoffe angesprochen (Art. 25). Trotz der nur allgemeinen Fassung dieser Vorschriften läßt sich ihnen doch entnehmen, daß weder das Ablassen von Öl bzw. von anderen umweltschädlichen Substanzen noch die Abfallbeseitigung auf Hoher See mit den ausdrücklich in Art. 2 des Übereinkommens über die Hohe See genannten Freiheitsrechten gleichzusetzen ist ${ }^{4}$. Auf der anderen Seite geben sie aber keinerlei wirkliche Handhabe, auf einen Schutz der maritimen Umwelt hinzuwirken.

Die erste detaillierte Konvention zum Schutze der Meere gegen Umweltschäden ist das von der IMCO initiierte internationale Übereinkommen zur Verhütung der Verschmutzung der See durch Öl, 1954, in der Fassung vom 11. April 19625. Es verbietet Tankern und nach einer Übergangszeit auch Frachtern einer besonders genannten Größe, Öl oder ölhaltige Gemische einer bestimmten Konzentration im Bereich der im Annex A genannten Verbotszonen abzulassen. Verbotszonen sind grundsätzlich alle an die Küsten angrenzenden Meeresgebiete bis zu $50 \mathrm{sm}$, für einige Küstenzonen $100 \mathrm{sm}$ oder sogar $150 \mathrm{sm}$. Jeder Staat hat nach diesem Übereinkommen das Recht, die Ausdehnung der vor seiner Küste liegenden Verbotszonen auf $100 \mathrm{sm} \mathrm{zu}$ fordern. Diese Erweiterung tritt 6 Monate nach der Erklärung in Kraft, wenn nicht ein anderer Vertragsstaat widerspricht ${ }^{6}$.

Um eine Kontrolle der Schiffe zu ermöglichen, wird die Führung eines Öltagebuches vorgeschrieben, in dem Tätigkeiten an Bord, die in aller Regel eine Umweltverschmutzung mit sich bringen, $z u$ verzeichnen sind. Das Öltagebuch kann von den Vertragsstaaten kontrolliert werden, wenn die Schiffe sich in ihren Häfen befinden. Werden dabei Vergehen festgestellt, so sind diese dem Flaggenstaat zu melden, der die Untersuchung und Bestrafung einleitet.

Das Konzept des Übereinkommens wurde in einer bislang noch nicht in Kraft getretenen Änderung von 1969 modifiziert, die das Prinzip der Verbotszonen aufgab. Hierin kommt zum Ausdruck, daß das Gebot des maritimen Umweltschutzes nur global zu lösen ist und sich nicht auf die küstennahen Meeresgebiete beschränkt. Die Änderung gestattet Tankern und sonstigen Handelsschiffen nur noch unter ganz bestimmten eng umgrenzten Voraussetzungen, Öl oder ölhaltige Gemische abzugeben für den Bereich der küstennahen Zonen wird ihnen dies außer in Notfällen - sogar vollständig untersagt (Art. III - V $)^{7}$.

\footnotetext{
4 Caflisch, Some Aspects of Oil Pollution from Merchant Ships, in: Annales D'Etudes Internationales 1973, Vol. 4, S. 213 (214) a. A. Dinstein, Oil Pollution by Ships and Freedom of the High Seas, in: Journal of Maritime Law and Commerce, Vol. 3 (1971/72), S. 363 (364 f.) Ehmer, Der Grundsatz der Freiheit der Meere und das Verbot der Umweltverschmutzung, Berlin 1974, S. $37 \mathrm{f}$.

5 BGBl. 1964 II, S. 749.

6 Nach Auskunft der IMCO sind bislang keine entsprechenden Erklärungen eingegangen - Schreiben des Legal Officer vom 17. 2. 1975.

7 Text der Änderung von 1969: ILM Vol. 9 (1970), S. 1. Zu den Schwierigkeiten, einen Verstoß gegen die Bestimmungen des Ubereinkommens festzustellen vgl. du Pontavice, Pollution, in: The Future of the Law of the Sea, Proceedings of the Symposium on the Future of the Law of the Sea at Den Helder by the Royal Netherlands Naval College and the International Law Institute of Utrecht State University 26 and 27 June 1972, The Hague 1973, S. 104 (118).
} 
Diese von der IMCO entwickelten Instrumente sind dadurch gekennzeichnet, daß sie auf dem sogenannten Flaggenstaatsprinzip beruhen, d. h. ihre Durchsetzung für den Bereich der Hohen See nur jeweils durch den Staat erfolgt, dessen Flagge das betreffende Schiff führt. Den Küstenstaaten werden außerhalb ihres Hoheitsgebietes weder Kontrollrechte noch Durchsetzungsbefugnisse zuerkannt. Die bislang genannten Übereinkommen schränken ihre Wirksamkeit dadurch ein, daß sie lediglich das bewußte Ablassen von Öl untersagen. Sie verzichten dagegen fast völlig auf die Einführung irgendwelcher Präventivmaßnahmen, die die Gefahr eines unabsichtlichen OOlablassens - sei es während des normalen Betriebes oder als Folge eines Schiffsunglücks - verringern könnten ${ }^{8}$.

Mit der Einführung präventiver Maßnahmen hat sich das Institut de Droit International auf seiner Tagung vom 4.-13. September 1969 beschäftigt. Es fordert Bau- und Ausrüstungsvorschriften sowie navigatorische Sicherheitsmaßnahmen, um die Gefahr von Schiffszusammenstößen sowie deren Folgeerscheinungen zu verringern. Bei den Beratungen kam zum Ausdruck, daß derartige Maßnahmen Einschränkungen für die Schiffahrt nach sich ziehen. Um diese nicht noch zu verstärken, wurde die Einführung von küstenstaatlichen Kontroll- und Durchsetzungsbefugnissen außerhalb ihrer Hoheitsgewässer abgelehnt ${ }^{9}$. Die in der Resolution des Institut de Droit International enthaltenen Anregungen griff die IMCO auf und legte 1971 ein Übereinkommen „Concerning Tank Arrangements and Limitation of Tank Size" als Ergänzung des Übereinkommens von 1954 vor ${ }^{10}$. Diese Ergänzung hat zum Ziel, die Folgen von Tankerunfällen zu verringern ${ }^{11}$. Ebenfalls sah die IMCO für 64 Seegebiete eine Einbahnregelung (Traffic Separation) vor, um die Kollisionsgefahr auf stark befahrenen Seewegen zu verkleinern ${ }^{12}$.

Eine wirkliche Neuorientierung der Aktivitäten der IMCO auf dem Gebiet des maritimen Umweltschutzes enthält erst die "Convention for the Prevention of Pollution from Ships“, die vom 15. 1.-31. 12. 1974 zur Unterzeichnung auslagi3. Sie behandelt nicht mehr allein die Meeresverschmutzung durch Öl, sondern allgemein durch schädliche Substanzen und erweitert damit ihren Wirkungskreis erheblich. Gleichzeitig nimmt sie das bis 1962 verfolgte Konzept des besonderen Schutzes der Küsten wieder auf, indem nunmehr in den küstennahen Zonen außer in Notfällen keinerlei Öl, ölhaltige Gemische oder sonstige schädliche Substanzen in das Meer eingebracht werden dürfen. Außerhalb der Verbotszonen ist das Ablassen von Öl nur unter besonders engen Voraussetzungen zulässig. Daneben sieht das Übereinkommen detaillierte schiffbauliche Sicherheitsvorkehrungen vor, die sowohl zur Verringerung der Folgen bei Schiffskollisionen dienen sollen, die aber auch die Aufgabe haben, zu verhindern, daß bei Betrieb der Schiffe Öl in die Bilgen gelangt und dann bei deren Reinigung unabsichtlich in das Meer gepumpt wird. Die Flaggenstaaten sind verpflichtet, durch eine Bauprüfung sowie durch periodische Kontrollen die Einhaltung dieser Sicherheitsvorschriften zu gewährleisten. Über die Inspektion werden Bescheinigungen erteilt, die von den übrigen Vertragsstaaten anzuerkennen sind.

8 Die Staaten werden einzig dazu verpflichtet, in ihren Häfen Einrichtungen vorzusehen, an die die Schiffe die angesammelten Altölrückstände abgeben können.

9 Andrassy, Etudes des mesures internationales les plus aptes à prévenir la pollution des milieux maritimes, in: Annuaire de l'Institut de Droit International (1969), Bd. 53, Teil I, S. 547 (568 f.).

10 Text in: ILM, Vol. 11 (1972), S. 267.

11 Sie erhielt bis zum Dezember 1974 allerdings erst 8 Ratifikationen.

12 RGDIP 1972, S. 530, vgl. dazu auch Doc. A/AC. 138/SC. III L. 30 Annex.

$13 \mathrm{Bis}$ zum Dezember 1974 erfolgte die Unterzeichnung von Dänemark, BRD, Italien, Schweden, UdSSR, Großbritannien, Australien, Brasilien, Irland, Niederlande, Frankreich, Bulgarien, Polen, DDR, Spanien und USA, Text: ILM, Vol. 12 (1973), S. 1319. 
Den Küstenstaaten hat die IMCO allerdings in keinerlei nennenswertem Umfang Kontrollbefugnisse eingeräumt, insoweit hält sie an dem bislang verfolgten Konzept fest. Die Küstenstaaten können lediglich die Öltagebücher sowie die Sicherheitszertifikate bei Schiffen prüfen, die sich in ihren Häfen aufhalten. Nur wenn der Zustand eines Schiffes oder seiner Ausrüstung eindeutig mit den Angaben der Sicherheitsbescheinigung nicht in Einklang steht, vermag der Küstenstaat die Weiterfahrt zu verhindern, bis gewährleistet ist, daß diese kein Sicherheitsrisiko mehr bedeutet. Bei einer Zuwiderhandlung gegen die Umweltschutzvorschriften im Bereich der Hohen See besteht für den feststellenden Staat allein die Möglichkeit, dies dem Flaggenstaat mitzuteilen, der dann die Bestrafung vornimmt und den Berichtsstaat entsprechend unterrichtet.

Sonderregelungen für ein beschränktes Eingreifen auf Hoher See bei Tankerunfällen enthält die "Convention Relating to Intervention on the High Seas in Cases of Oil Pollution Casualities“, $19699^{14}$ sowie das "Protocol Relating to Intervention on the High Seas in Cases of Marine Pollution by Substances others than Oil“, 197315. Danach können bei Tankerunfällen Maßnahmen auf Hoher See gegen fremde Schiffe ergriffen werden, um eine bevorstehende schwere Gefährdung der Küste zu verhindern. Die Konvention enthält weitere prozedurale Vorschriften in bezug auf Konsultationspflichten sowie den Hinweis darauf, daß jede der ergriffenen Maßnahmen auf ihre Verhältnismäßigkeit zu prüfen ist. Im Grunde genommen bedeuten diese Übereinkommen nicht mehr als eine Ausprägung des völkergewohnheitsrechtlich anerkannten Notrechts ${ }^{16}$.

Keine eigentliche Ausdehnung der küstenstaatlichen Befugnisse erlaubt dagegen das sogenannte Bonn-Abkommen ${ }^{17}$. Die Nordseeanrainerstaaten erhalten lediglich bestimmte Überwachungszonen und verpflichten sich, bei der Bekämpfung der Ölverschmutzung aktiv zusammenzuwirken.

Eine Sonderregelung existiert für die Abfallbeseitigung auf Hoher See. Diese Frage wirft offenbar weniger tiefgreifende rechtliche Probleme auf, vor allem sind keine wesentlichen Einschränkungen der Schiffahrtsfreiheit zu befürchten. Die Oslo "Convention for the Prevention of Marine Pollution by Dumping from Ships and Aircraft" vom 15. Februar 1972 enthält eine Liste von Stoffen (Annex I), die überhaupt nicht sowie eine weitere Aufzählung von Substanzen (Annex II), die nur nach Genehmigung im Nordostatlantik und in dessen Randmeeren versenkt werden dürfen. Die Einhaltung und die Durchführung dieses Übereinkommens wird durch eine ständige Kommission überwacht (Art. $16 \mathrm{ff}$.). Insofern liegt hier erstmalig die Verantwortung für den Umweltschutz nicht mehr allein bei den jeweiligen Vertragstaaten, was ohne Zweifel eine Neuerung bedeutet. Allerdings stehen der Kommission keine wirklichen Durchsetzungsbefugnisse zu, da sie sich auf Empfehlungen beschränken muß (Art. 18). Materiell schärfere Anforderungen enthält die London "Convention on the Prevention of Marine Pollution by Dumping of Wastes and other Matters", 197218, da sie nicht nur die

14 Text in: ILM, Vol. 9 (1970), S. 25.

15 Text in: ILM, Vol. 13 (1974), S. 605. Obwohl beide Ubereinkommen noch nicht in Kraft getreten sind, hat Großbritannien die Rechtsgrundlage für ein entsprechendes Tätigwerden geschaffen: The Oil in Navigable Waters (Shipping Casualities) Order 1971 (ILM Vol. 11 (1972), S. 173); Oil in Navigable Waters Act 1971 (ILM Vol. 10 (1971), S. 584.

16 Schultheis: Umweltschutz und die Freiheit der Meere, Bonn 1974, S. $198 \mathrm{f}$; Caflisch, International Law and Ocean. Pollution: The Present and the Future, in: Revue belge de droit international 1972, S. 7.

17 Ưbereinkommen zur Zusammenarbeit bei der Bekämpfung von Olverschmutzungen in der Nordsee vom 9. Juni 1969 (BGBl. 1969 II, S. 2066.

18 Text der Oslo-Konvention: ILM, Vol. 11 (1972), S. 262; London-Konvention: ILM, Vol. 11 (1972), S. 1291. 
Ablagerung einiger Stoffe verbietet, sondern auch im übrigen die Abfallbeseitigung auf See von einer Genehmigung abhängig macht. Die Durchführung dieser Konvention liegt allerdings ausschließlich in den Händen der Vertragstaaten. Sie sind zuständig für die Genehmigungen der Beseitigung von Stoffen, die in ihrem Hoheitsgebiet geladen werden oder von einem Schiff bzw. Flugzeug versenkt werden sollen, das ihre Nationalität besitzt (Art. VI).

Keine eigene Konvention wurde bislang für die Bekämpfung der maritimen Umweltverschmutzung von Land aus entwickelt, obwohl von daher nach einer Studie der USA der größte Teil der Meeresverschmutzung stammt ${ }^{19}$. Die Entwicklung allgemein gültiger Standards dürfte hier auch schwerfallen, da sie, um effektiv zu sein, tief in die Umweltschutzprogramme der einzelnen Staaten eingreifen müßten. Die von der Stockholmer Umweltschutzkonferenz 1972 vorgelegte Erklärung beschäftigt sich zwar mit diesem Problem, hat jedoch keine verbindliche Wirkung. Sie verlangt lediglich, daß die Staaten schädliche Umwelteinflüsse aus ihrem Hoheitsgebiet verhindern (Prinzip 21, 22). Gleichzeitig werden aber den Entwicklungsländern Sonderrechte zugebilligt, um nicht durch zu strikte Umweltschutzforderungen deren Volkswirtschaft übermäßig zu belasten bzw. den Aufbau einer Industrie zu behindern. (Prinzipien 10, 11, 12, 23) ${ }^{20}$. Die Aufnahme dieser Sonderbestimmungen zeigt deutlich die wirtschaftlichen Implikationen eines derartigen Umweltschutzprogramms.

\section{Nationale Maßnahmen}

Obwohl die genannten internationalen Übereinkommen zum Schutz der maritimen Umwelt von vielen Kritikern als nicht ausreichend angesehen werden, sind nur eine kleine Zahl von Staaten dazu übergegangen, einseitig Maßnahmen zu ergreifen. Das mag daran liegen, daß eine Reihe von Staaten ihre Küstengewässer ausgedehnt bzw. Fischereischutzzonen errichtet haben und in diesem Raum auch Umweltschutzbefugnisse beanspruchen. Hier fehlt ein Bedürfnis für isolierte nationale Umweltschutzkompetenzen auf Hoher See.

Bei der Untersuchung nationaler Maßnahmen sind daher folgende Typen zu unterscheiden: Die Ausübung nationaler Kompetenzen durch Erweiterung der Küstengewässer, Schaffung echter Umweltschutzzonen auf Hoher See, in denen der Küstenstaat Sonderrechte ausübt, die Umweltschutzregelung im Rahmen der Errichtung von Fischereischutzzonen, Umweltschutzvorschriften bei Ausbeutung des Festlandsockels und schließlich die Durchführung der IMCO-Übereinkommen.

a) Erweiterung der Küstengewässer.

Brasilien ${ }^{21}$, wie auch unter anderem Ekuador ${ }^{22}$, Peru ${ }^{23}$ und Panama ${ }^{24}$ beanspruchen Küstengewässer von $200 \mathrm{sm}^{25}$ - es fehlen bei den letztgenannten im Gegensatz zu Brasilien aber eigene Umweltschutzmaßnahmen.

Brasilien gestattet gem. Art. 3 Schiffen aller Staaten die friedliche Durchfahrt durch seine erweiterten Küstengewässer, dabei haben sie aber die brasilianische

\footnotetext{
19 Doc. A/AC. 138/SC. III/L. 36.

20 Stockholm Declaration, in: Lay-Churchilll-Nordquist; New Directions in the Law of the Sea, Documents Vol. II. New York, London 1973, S. 712.

21 Decree Law No. 1098 vom 25. 3. 1970, ST/LEG/SER.B/16, S. 3.

22 Civil Code vom 20. 8. 1960, in: ST/LEG/SER.B/15, S. 78.

23 Gesetzesdekret vom 24. 7. 1969, in: ST/LEG/SER./B/16, S. 28

24 Act No. 31 vom 2. 2. 1967, in: ST/LEG/SER.B/15, S. 105.

$25 \mathrm{Vgl}$. zur Ausdehnung der Küstengewässer im übrigen: Kehden, Die Inanspruchnahme von Meereszonen und Meeresbodenzonen durch Küstenstaaten, 2. Aufl. Hamburg 1971.
} 
Gesetzgebung zum Schutz der maritimen Umwelt zu beachten (Art. 3, \2). Diese $^{26}$ verbietet unter Strafandrohung die Ablagerung schädlicher Stoffe, wie auch die Verunreinigung durch Wartungsmaßnahmen bei Schiffen. Diese Lösung ist in sich konsequent. Zwar kann man bislang nicht von einer völkerrechtlichen Anerkennung eines derartig breiten Küstengewässers sprechen, es ist aber nicht zu leugnen, daß der Küstenstaat im Bereich seiner Küstengewässer nach derzeit geltenden Vorstellungen die Möglichkeit hat, Vorschriften im Rahmen des Umweltschutzes zu erlassen und auch durchzusetzen, um so die Erhaltung seiner Küsten zu gewährleisten. Immerhin hat sich Brasilien mit Verunreinigungsverboten begnügt und bislang auf die Einführung präventiver Maßnahmen, die in aller Regel besondere Belastungen für die Schiffahrt enthalten, verzichtet.

b) Umweltschutzzonen

Den Weg, anschließend an die eigenen Küstengewässer eine Umweltschutzzone zu errichten, in der dem Küstenstaat ausschließliche Regelungs- und Kontrollbefugnisse zustehen, hat Kanada gewählt ${ }^{27}$. Vergleichbar damit, zumindest was die Auswirkungen auf die Schiffahrt betrifft, sind die Regelungen der UdSSR für den nördlichen Seeweg ${ }^{28}$ und die Gesetzgebung von Massachusetts ${ }^{29}$. Ahnliche Regelungen sicht auch Ghana vor, das sich die Errichtung einer an seine Küstengewässer angrenzenden Schutzzone mit unbeschränkter Breite vorbehält ${ }^{30}$ - Honduras nimmt eine Schutz- und Überwachungszone von $200 \mathrm{sm}$ in Anspruch, ohne das Ausmaß seiner Kompetenzen zu fixieren ${ }^{31}$ - die USA verbieten das Ablassen von $\mathrm{O}$ il im Bereich einer Anschlußzone gem. Art. 24 der Konvention über das Küstenmeer und die Anschlußzone ${ }^{32}$.

Das kanadische Gesetz schützt Teile des Atlantiks und des Nordpolarmeeres vor der kanadischen Ostküste. Das Gebiet wird durch $60^{\circ}$ nördlicher Breite und $141^{\circ}$ westlicher Länge sowie eine Linie, die in $100 \mathrm{sm}$ Abstand von dem kanadischen Land verläuft, begrenzt. Soweit sich das Gesetz auf den Meeresbergbau und seine Auswirkungen auf die Umwelt bezieht, gehört das Wasser über dem kanadischen Festlandsockel mit in den Schutzbereich des Gesetzes; wegen dessen Ausdehnung wird die 100-sm-Begrenzung teilweise überschritten. Das Gesetz verbietet allgemein das Einleiten von schädlichen Abfallstoffen jeder Art von Schiffen wie auch von Land aus. Es sieht Maßnahmen bei Schiffskollisionen und Havarien vor und regelt die Fragen der Schadensersatzpflicht. Besondere Bedeutung kommt der Einführung präventiver Maßnahmen zu, durch die das Entstehen einer Ölverschmutzung verhindert werden soll. So kann der Governor in Council jedes Gebiet der arktischen

26 Act No. 5357 vom 17. 11. 1967, Decree No. 50877 vom 29. 6. 1961, ST/LEG/SER.B/16, S. 64.

27 Arctic Waters Pollution Prevention Act, 1970, Text: ILM, Vol. 9 (1970), S. 543. Vgl. dazu: Morin, Le progrès technique, la pollution et l'évolution récente du droit de la mer au Canada, particulièrement à l'égard de l'Arctique, in: Annuaire Canadien de Droit International 1970, S. 158; Pharand, Oil Pollution Control in the Canadian Arctic, in: Texas International Law Journal, Vol. 7 (1971), S. 45, Schultheiss a. a. O.

28 Statute of the Administration of the Northern Sea Route Attached to the Ministry of the Maritime Fleet, Text in: ILM, Vol. 11 (1972), S. 645. Vgl. dazu Butler, Pollution Control and the Soviet Arctic, in: ICLQ Vol. 21 (1972), S. 557; Dehner, Creeping Jurisdiction in the Arctic: Flas the Soviet Union Joined Canada, in: Harvard International Law Journal Vol. 13 (1972), S. 271.

29 An Act Relative to the Territorial Waters of the Commonwealth and extending the authority of the director of the division of marine fisheries, in: VRU 1973, S. 242 mit Einführung von Wedel, Einseitige Ausdehnung der Souveränitätsrechte auf das Hohe Meer, in: VRU, 1973, S. 235.

30 The Territorial Waters and Continental Shelf Act 1963, vom 19. 4. 1963, in: ST/LEG/SER.B. 15, S. 85 .

31 Congressional Decree No. 25 vom 17. 1. 1951, in: ST/LEG/SER./B/6, S. 22.

32 Federal Water Pollution Control Act, as amended up to 1970, ST/LEG/SER.B/16, S. 71. 
Gewässer zur Sicherheitszone erklären und weitreichende Regelungen für den Schiffsverkehr in ihnen erlassen. Vor allem hat er die Befugnis, die Sicherheitszonen für solche Schiffe zu sperren, die nicht den von ihm erlassenen Sicherheitsbestimmungen hinsichtlich der Konstruktion des Schiffskörpers, der Tanks, der Antriebs- und Steueranlagen, der Art der Ladung sowie navigatorischen Ausrüstung und der Zahl und Ausbildung des seemännischen Personals entsprechen. Er kann das Befahren der Sicherheitszonen von der Inanspruchnahme eines Lotsen abhängig machen oder die Region für den Schiffsverkehr völlig sperren. Die Einhaltung der Vorschriften wird durch sog. "Pollution Prevention Officers" überwacht, die mit weitreichenden Prüfungsbefugnissen ausgestattet sind.

Vergleichbaren Einschränkungen unterliegt die Ausbeutung der Rohstoffe im Festlandsockelbereich sowie die Errichtung, Anderung oder Erweiterung von Anlagen auf Inseln und dem kanadischen Festland, soweit damit die Gefahr einer Umweltverschmutzung verbunden ist. Die Baupläne sind dem Governor in Council vorzulegen; er kann mit Rücksicht auf den Umweltschutz deren Änderung verlangen oder ihre Ausführung gänzlich verbieten.

Die Problematik derartiger Kontrollzonen liegt darin, daß sich durch sie, entgegen dem gegenwärtig, noch geltenden, Seerecht die Jurisdiktion eines Küstenstaates auch auf Teile der Hohen See erstreckt. Schiffe fremder Nationalität werden damit Einschränkungen unterworfen, denen sie sonst nur im Bereich der Küsten- und Binnengewässer unterliegen. Auf der Hohen See unterstehen Schiffe dagegen - sieht man einmal von den Sanktionsmöglichkeiten gegenüber Piraten und Sklavenhandel ab - allein der Hoheitsgewalt des Flaggenstaates. Insofern bedeutet die Einführung einer derartigen Kontrollzone einen Bruch mit diesem auch in dem Genfer Übereinkommen über die Hohe See verankerten Prinzip.

c) Umweltschutz im Bereich von Fischereischutzzonen.

Entschieden weniger einschneidend für die Schiffahrt ist die Praxis, Umweltschutzgebote im Zusammenhang mit der Errichtung von Fischereischutzzonen $\mathrm{zu}$ erlassen. Derartiges sehen die Gesetze von Brasilien ${ }^{33}$, Ekuador ${ }^{34}, \mathrm{Kuba}^{35}$, Indien ${ }^{36}$, Sri Lanka ${ }^{37}$ und Kanada ${ }^{38}$ vor. Sie beschränken sich allerdings darauf, das Einleiten von für den Fischbestand schädlichen Substanzen zu untersagen oder sogar nur ganz allgemein Erhaltungsmaßnahmen vorzusehen, so daß es der Exekutive überlassen bleibt, entsprechende Umweltschutzvorschriften zu konkretisieren.

d) Umweltschutz bei Ausbeutung des Festlandsockels.

Nicht wesentlich detaillierter sind die Regelungen der Staaten, die für Ausbeutungsmaßnahmen in ihrem Festlandsockelbereich Regelungen zum Schutze der maritimen Umwelt erlassen haben. Sie beschränken sich wie z. B.

\footnotetext{
33 Legislative Decree Nr. 221 vom 28. 2. 1967 on Fishing, in: ST/LEG/SER.B/16, S. 267.

34 Decree No. 991 vom 23. 5. 1961 (Art. 50), in: ST/LEG/SER.B/15, S. 631

$35 \mathrm{Kuba}$ beansprucht eine Schutzzone zur Erhaltung der Meeresreichtümer ohne räumliche Begrenzung und ohne nähere Angabe der Regelungsinhalte, Legislative Decree No. 1948 of 25 January 1955 relating to the Territorial Sea, in: ST/LEG/SER.B/6, S. 8.

36 Presidential Proclamation of 29 November 1956, on Conservation Zones, in: ST/LEG/SER.B/16,

37 Proclamation of 19 December 1957 by the Governor General on the Rights over the Continental Shelf and Conservation Zones, in: ST/LEG/SER.B/16, S. 318.

38 Fisheries Act, 1952, as amended up to 1970, in: ST/LEG/SER.B/16, S. 273.
} 
Fidschi $^{39}$, Bahamas ${ }^{40}$, Malta ${ }^{41}$ und Madagaskar ${ }^{42}$ darauf, das Auslaufen von Öl aus Ölleitungen oder während der Bohrarbeiten zu verbieten und unter Strafe zu stellen oder die Eigentümer dazu zu verpflichten, etwaige Verunreinigungen zu beseitigen, Thailand ${ }^{43}$.

e) Die Übernahme der IMCO-Konvention.

Die meisten der Staaten, die Regelungen zum Schutz der maritimen Umwelt kennen, haben sich darauf beschränkt, die von der IMCO erarbeiteten Übereinkommen inhaltlich in nationales Recht zu übertragen. Dennoch bestehen in dieser Hinsicht im einzelnen nicht unerhebliche Unterschiede. Sie rühren im wesentlichen daher, daß die Gesetze teilweise Sonderregelungen für die Küstengewässer wie für die einheimische Schiffahrt enthalten. Weitere Differenzen ergeben sich daraus, daß vereinzelt das Übereinkommen in der Fassung von 1969 in innerstaatliches Recht übernommen wurde.

Als exemplarisch sei hier in erster Linie die Regelung von Ghana ${ }^{44}$ herausgegriffen. Sie richtet sich, soweit sie nicht die Hoheitsgewässer von Ghana betrifft, nur an Schiffe unter der Flagge von Ghana. Ihnen wird verboten, Öl oder ölhaltige Flüssigkeitsgemische einer bestimmten Konzentration im Bereich der im Gesetz genannten Sperrzonen abzulassen. Diese Sperrzonen entsprechen im wesentlichen den Vorschriften des Übereinkommens über die Verhütung der Verschmutzung auf See durch Öl in der Fassung von 1962. Außerdem wird der zuständige Minister ermächtigt, weitere Teile des Meeres außerhalb der Hoheitsgewässer von Ghana zur Verbotszone zu erklären. Damit soll wohl der in Annex A, Abs. 3 des genannten Übereinkommens aufgezeigten Möglichkeit einer Erweiterung der Schutzzonen Rechnung getragen werden. Weitergehend sind die Vorschriften für die ghanesischen Hoheitsgewässer. Hier ist es allen Schiffen untersagt, Öl abzulassen. Vergehen dagegen werden unter Strafe gestellt. Als besondere Vorsichtsmaßnahme ist es Schiffen untersagt, nachts Öl zu übernehmen. Hinsichtlich der Bauvorschriften beschränkt sich die ghanesische Regelung auf die Wiederholung der in Art. 7 des Übereinkommens genannten Modalitäten.

Demgegenüber sind die Bauvorschriften von Neuseeland ${ }^{45}$, dessen Regelung sonst denen von Ghana gleicht, und von Spanien ${ }^{46}$ detaillierter.

Eine Besonderheit weist die Gesetzgebung von Australien ${ }^{47}$ auf, wenn sie auch im übrigen dem aufgezeigten Grundmuster folgt. Danach stehen den australischen zuständigen Stellen wesentliche Inspektionsrechte zu; sie können bei ausländischen Schiffen, die unter der Flagge eines fremden Staates fahren, in vollem Umfang überprüfen, ob dieses Schiff den Übereinkommen der IMCO oder der entsprechenden Gesetzgebung des Flaggenstaates entspricht.

Weitergehende Inspektionsrechte erlaubt auch die Gesetzgebung von Singapur ${ }^{48}$. Danach haben die zuständigen Stellen das Recht, jedes ausländische Schiff im

39 Continental Shelf Act, 1970, in: ST/LEG/SER.B/16, S. 141.

40 Continental Shelf Act, 1970, in: ST/LEG/SER.B/16, S. 172

41 Continental Shelf Act, 1966, in: ST/LEG/SER.B/16, S. 156.

42 Loi No. 70 - 016, 15. 7. 1970 Portant Réglementation maritime des Installations et autres Dispositifs sur le Plateau Continental, in: ST/LEG/SER.B/16, S. 152.

43 Petroleum Act, 1971, in: ST/LEG/SER.B/16, S. 99.

44 Oil in Navigable Waters Act, 1964, in: ST/LEG/SER.B/15, S. 489.

45 Oil in Navigable Waters Regulations, 1971, in: ST/LEG/SER.B/16, S. 221

46 Order of the Council of Ministers of 1 June 1963 establishing the rules to be followed in the building of new ships in order to prevent pollution of the sea by oil, in: ST/LEG/SER.B/16, S. 236.

47 Pollution of the Sea by Oil Regulations as amended up to 1967 in: ST/LEG/SER.B/16, S. 181; Pollution of the Sea by Oil Act (No. 4 of $1965 ; 12.4 .1965$ ), in: ST/LEG/SER.B/15, S. 477.

48 Prevention of Pollution of the Sea Act, 1971, in: ST/LEG/SER.B/16, S. 224. 
Hafen festzuhalten, wenn ein vernünftiger Grund für die Annahme besteht, daß dieses Schiff in den Hoheitsgewässern von Singapur OOl abgelassen hat oder daß Ơl auf Hoher See abgegeben wurde und dieses in die Hoheitsgewässer Singapurs treibt. Abgesehen von einer möglichen strafrechtlichen Verfolgung ist der Schiffseigner zu Schadenersatz verpflichtet.

\section{Maritimer Umweltschutz nach den Vorschlägen im Meeresbodenausschuß und der Seerechtskonferenz}

Die mit der Regelung des maritimen Umweltschutzes verbundenen Schwierigkeiten traten erst im Verlauf der Arbeiten des Meeresbodenausschusses und der Seerechtskonferenz deutlich zu Tage. Vor allem wurde die Diskussion im Meeresbodenausschuß anfangs dadurch erschwert, daß man nicht hinsichtlich der einzelnen Verschmutzungsquellen unterschied. Inzwischen hat sich die Ansicht durchgesetzt, daß die einzelnen Verschmutzungsursachen verschiedenartige Maßnahmen erfordern ${ }^{49}$. Bislang kam eine Einigung nur über die folgenden Grundsätze zustande: Allgemeine Verpflichtung der Staaten, die Meeresumwelt zu erhalten, das Verbot des Verschmutzungstransfers und die Pflicht der Staaten, bei der Bekämpfung der Umweltverschmutzung einander technische Hilfe zu leisten ${ }^{50}$. Überschattet wurde die gesamte Diskussion von dem Verlangen einer Reihe von Entwicklungsländern, bei einer internationalen Regelung des Umweltschutzes auf ihre Leistungsfähigkeit Rücksicht zu nehmen. Sie fordern eine teilweise Freistellung von den aus internationalen Regelungen resultierenden Pflichten und somit die Möglichkeit, an Anlagen und Schiffen, die ihrer Jurisdiktion unterliegen, geringere Anforderungen stellen zu können ${ }^{51}$.

Insgesamt scheint der Teilaspekt der Umweltgefährdung vom Festland aus keine speziellen Probleme aufzuwerfen. Man ist sich im Grunde genommen weitgehend darüber einig, daß die Regelungs- und Durchsetzungskompetenzen bei den betroffenen Staaten selbst - also in aller Regel bei den Küstenstaaten liegen sollen. Unklarheit herrscht lediglich über eine mögliche Bindung des nationalen Gesetzgebers an international oder regional entwickelte Mindestanforderungen ${ }^{52}$. Entsprechendes gilt für den Meeresbergbau innerhalb der Meereszone, die nationaler Kontrolle unterliegt und die damit verbundenen Gefahren für die Meeresumwelt. Auch hier ist im wesentlichen unumstritten, daß die Regelungs- und Durchsetzungskompetenzen dem Küstenstaat zustehen, wobei die Frage nach einer Bindung an internationale Standards ebenfalls noch offen ist ${ }^{53}$. Der Umweltschutz bei der Ausbeutung des Tiefseebodens soll nach einhelliger Meinung in die Kompetenz der neu zu schaffenden Meeresbergbaubehörde fallen ${ }^{54}$. Finsichtlich der Abfallbeseitigung auf See zeichnet sich die Tendenz ab, die Befugnisse der Küstenstaaten zu erweitern ${ }^{55}$. Die eigentlichen Probleme wirft dagegen die $\mathrm{Be}-$ kämpfung der Meeresverschmutzung durch Schiffe auf. Hier stehen sich, wie auch

49 Doc. A/Conf. 62/C.3/L. 14.

50 Doc. A/Conf. 62/C. 3/L. 15.

51 So z. B. Indien

52 WG 2/Paper No. 15, in: Doc. A/9021 Vol. I, S. 93, Doc. CRP/MP./9, Sec. I.

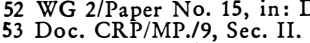

54 Doc. CRP/MP./9, Sec. II.

55 Doc. CRP./MP./12/Rev. 1; Doc. A/Conf. 62/C. 3/SR. 5, S. 17. 
schon in der bisherigen Staatenpraxis feststellbar, die Befürworter einer Zonenlösung und die Vertreter des Flaggenstaatsprinzips mit einer Fülle von Vorschlägen gegenüber. Die Vorschläge lassen sich im wesentlichen den folgenden Grundtypen zuordnen.

a) Ausdehnung der Küstengewässer.

Am weitesten gehen in jeder Hinsicht die Ansprüche derjenigen Staaten, die eine Erweiterung ihrer Küstengewässer bis auf $200 \mathrm{sm}$ fordern. (So z. B. ausdrücklich Uruguay ${ }^{56}$, Brasilien ${ }^{57}$, Ekuador, Panama, Peru ${ }^{58}$.) Denn im Bereich der Küstengewässer hätten die Staaten nach dem überkommenen Konzept das Recht, Regelungen zum Schutze der maritimen Umwelt zu erlassen und auch durchzusetzen.

Eine Sonderstellung wegen seiner Detailliertheit nimmt insofern der Vorschlag Maltas ein ${ }^{59}$. Nach Art. 36 des Malta-Entwurfs übt der Küstenstaat seine Hoheitsgewalt bis zu $200 \mathrm{sm}$ Entfernung von seiner Küste aus. Es handelt sich hier wegen seiner starken Bindung an das Völkerrecht allerdings nicht um Küstengewässer im eigentlichen Sinne, wie sie in der Konvention über die Küstengewässer und die Anschlußzone definiert werden, sondern nur um Teile der See, die unter nationaler Kontrolle stehen ("national ocean space“ - Art. 1 des Entwurfs). In diesem Bereich hat der Staat das Recht, Regelungen zum Schutze der Umwelt zu erlassen, die den entsprechenden Regeln des Völkerrechts über Schiffsverkehr und die Verhütung der Verschmutzung der Meere entsprechen sollen. Diese Vorschriften dürfen allerdings nicht diskriminierend wirken und die Schiffahrt nicht unbillig beeinträchtigen. Inwieweit dem Genüge getan worden ist, entscheidet im Zweifelsfall der Internationale Meeresgerichtshof. Die Überwachung der Seegebiete außerhalb der Hoheitsgewässer erfolgt durch eine internationale Behörde.

b) Umweltschutzzonen

Fast ebensoweit gehen diejenigen Staaten, die die Errichtung einer maritimen Umweltschutzzone fordern, in der dem Küstenstaat sowohl die Regelungsbefugnis wie auch die Kontroll- und Durchführungsbefugnisse zustehen. Dieses Konzept unterstützen neben Kanada, das insoweit versucht, seine arktische Umweltschutzzone völkerrechtlich abzusichern, im Meeresausschuß weitere 12 Staaten, wobei allerdings die küstenstaatlichen Befugnisse hinsichtlich Regelungs- und Durchsetzungsbefugnisse ausdrücklich offen gelassen wurden ${ }^{60}$. Am detailliertesten ist der Einzelvorschlag Kanadas ${ }^{61}$. Danach haben die Staaten die Möglichkeit, Umweltschutzzonen angrenzend an ihre Küstengewässer zu errichten, in denen ihnen neben dem Flaggenstaat die Durchsetzung völkerrechtlicher Umweltschutzvorschriften obliegt (Art. 10). Soweit allerdings die lokalen geographischen oder ökologischen Eigenheiten des Gebietes es erfordern, sollen sie auch das Recht haben, eigene Maßnahmen zur Sicherung der Umwelt zu ergreifen (Art. 4). Diese können sowohl die Kontrolle von Ablagerungen, die Regelung von Ausstattung, Ausrüstung und Kontrolle von Schiffen sowie von Anlagen des Meeresbergbaus umfassen (Art. 1). Es

56 Doc. A/AC. 138/SC. II/L. 24.

57 Doc. A/AC. 138/SC. II/L. 25; Doc. A/Conf. 62/C. 2/L. 10 und L. 17.

58 Doc. A/AC. $138 /$ SC. II/L. 27

59 Doc. A/AC. 138/53 A/AC. 138/SC. III/L. 33.

60 Doc. A/AC. 138/SC. III/L. 56.

61 Doc. A/AC. 138/SC. III/L. 28, 37, 37/Add. 1. 
wird lediglich gefordert, daß derartige Maßnahmen dem Umweltschutz dienen und nicht diskriminierend wirken.

c) Umweltschutz im Rahmen der Wirtschaftszonen.

$\mathrm{Zu}$ einem ähnlichen Ergebnis kommen in aller Regel diejenigen Staatenvorschläge, die von der Errichtung einer Wirtschaftszone ausgehen. Unterschiede ergeben sich lediglich dann, wenn die Schiffahrt von der nationalen Unweltschutzregelung ausgenommen wird oder in gewissem Umfang einer entsprechenden Bindung an die internationalen Regelungen unterliegt. Wenig differenziert ist in dieser Hinsicht der Vorschlag Kenias ${ }^{62}$, wonach bei dem Erlaß nationaler Regelungen zum Schutze der Umwelt im Bereich der Wirtschaftszonen die internationalen Konventionen sowie entwickelte Standards ebenso wie die geographischen und ökologischen Situationen zu berücksichtigen sind.

Dagegen greift der Vorschlag von 10 Staaten $^{63}$ auf der Seerechtskonferenz das bereits in der Staatenpraxis festgestellte Prinzip auf, bei der Regelung der Kompetenz zwischen der Verschmutzung von Land und Tätigkeiten im Meeresbergbau gegenüber denen der Schiffahrt zu unterscheiden. Er geht von dem Grundsatz aus, daß die Staaten verpflichtet sind, jede Beeinträchtigung der Umwelt durch Tätigkeiten im Bereich ihrer Jurisdiktion $\mathrm{zu}$ verhindern (Art. III). Es wird aber insoweit zwischen den Umwelteinflüssen von Land aus und bei Ausbeutung des Meeresbodens und der Verschmutzung der Schiffe unterschieden. Denn in der ersten Gruppe sind die völkerrechtlichen Regelungen und Standards lediglich zu berücksichtigen (Art. VII, 3a), während die Regelungen für Schiffe mit den völkerrechtlichen Normen und Standards übereinzustimmen haben. Allerdings wird dem Küstenstaat die Befugnis eingeräumt, auch in dieser Hinsicht strengere eigene Regelungen zu erlassen, soweit sie vernünftig sind und nicht diskriminierend wirken, und dies durch die besondere ökologische Verletzbarkeit des betreffenden Gebietes gefordert wird. Derartige Maßnahmen sind der zuständigen internationalen Behörde ohne Verzug mitzuteilen.

Wenn auch auf diese Art und Weise eine weitgehende Annäherung an die reinen Umweltschutzzonenvorschläge erfolgt ist, so wirkt jedoch die Einschränkung der Schiffahrt nach diesem Vorschlag weniger einschneidend, da zumindest, wenn auch in minimalem Umfang, die küstenstaatlichen Regelungsbefugnisse begrenzt sind.

In der Bindung der nationalen Rechtsgesetzbefugnis an internationale Richtlinien unterscheiden sich auch die übrigen Varianten dieses Grundkonzepts.

So sollen z. B. nach dem Vorschlag Australiens ${ }^{64}$ die Vernünftigkeit der Maßnahmen in erster Linie anhand der völkerrechtlichen Regelungen geprüft werden. Nach dem Vorschlag der $\mathrm{USA}^{65}$ hat der Küstenstaat dagegen im Bereich der Wirtschaftszonen nur das Recht, Unweltschutzmaßnahmen hinsichtlich des Meeresbergbaus zu erlassen. Die Verhütung der Meeresverschmutzung durch Schiffe verbleibt weitestgehend in der Kompetenz des Flaggenstaates (Art. VII). Der Küstenstaat hat echte Durchsetzungsmöglichkei-

62 Doc. A/AC. 138/SC. III/L. 41; Doc. A/Conf. 62/C. 3/L. 2.

63 Doc. A/Conf. 62/C. 3/L. 3 (Kanada, Fidschi, Ghana, Guyana, Island, Indien, Iran, Neuseeland, Philippinen).

64 Doc. A/AC. 138/SC. III/L. 27

65 Doc. A/AC. 138/SC. III/L. 36, 40. 
ten nur bei Schiffen, die seine Häfen anlaufen. Hier kann er allerdings alle Vergehen verfolgen, gleichgültig, wo sie begangen sind, wenn das Vergehen nicht länger als drei Jahre zurückliegt und er hierzu durch die anderen Staaten ermächtigt ist. Außerdem hat nach diesem Konzept jeder Staat für die auf Hoher See begangenen Verstöße ein Anzeigerecht. Die Bestrafung der Schiffseigner bzw. des Schiffskapitäns kann dann entweder der Flaggenstaat oder der Hafenstaat vornehmen, und zwar der Staat des Hafens, den das Schiff innerhalb von 6 Monaten anläuft. Eine weitgehend vermittelnde Haltung nimmt der Vorschlag Italiens ${ }^{60}$ ein, indem er versucht, die mit einer nationalen Errichtung der Umweltschutzzonen verbundenen Härten zu vermeiden. Danach kann nämlich eine derartige Zone nur von einer internationalen Behörde auf Antrag des Küstenstaates eingerichtet werden. Die Behörde erläßt dann die Normen, die dem Umweltschutz in dieser Zone dienen.

d) Trennung von Regelungs- und Durchsetzungsbefugnissen.

Von einer ganz anderen Konzeption gehen die Vorschläge der Niederlande ${ }^{67}$ und Frankreichs ${ }^{68}$ und - beschränkt auf die Meeresverschmutzung der Schiffe - der bereits erwähnte Vorschlag der USA aus. Bei ihnen erfolgt eine Trennung zwischen Rechtsetzungs- und Durchsetzungsbefugnis. Die Rechtsetzungsbefugnis - insbesondere hinsichtlich von Bau- und Ausrüstungsvorschriften - erfolgt durch völkerrechtliche Úbereinkommen, so daß insoweit ein allgemeiner Standard gesichert bleibt. Den Küstenstaaten obliegt allgemein für das an ihre Küstengewässer angrenzende Seegebiet (so Frankreich) oder aber bei Schiffen, die ihre Häfen anlaufen (Niederlande, USA), gegenüber allen Verstößen im Rahmen einer zeitlichen Begrenzung die Kontrollund Strafbefugnis. Diese Lösung vermeidet eine Rechtszersplitterung, die bei der Verwirklichung des kanadischen Vorschlags bzw. des Küstenmeerkonzepts die zwangsläufige Folge wäre, erlaubt aber eine effektive Uberwachung. Besonders scharf ist in dieser Hinsicht der Vorschlag der USA, wonach es das Recht des Küstenstaates ist, nach einem entsprechenden Verfahren gegenüber Schiffen eines Staates, der dauernd gegen die Umweltschutzvorschriften verstößt, Sondermaßnahmen zu ergreifen (Art. XIV).

e) Flaggenstaatsprinzip

Dem traditionellen Konzept folgt dagegen der Vorschlag der BRD ${ }^{69}$, der auf dem Flaggenstaatsprinzip beruht und insofern die von dem IMCO-Úbereinkommen vorgezeichnete Linie weiter verfolgt. Er sieht im Grunde genommen eine Dreiteilung der Kompetenzen vor und strebt auf diese Weise einen Ausgleich zwischen den Interessen der Küstenstaaten und denen der Schifffahrtsnation an. So liegt die Regelungsbefugnis für die Hohe See ausschließlich bei der internationalen Staatengemeinschaft, während sich die Küstenstaaten und die Flaggenstaaten die Kompetenzen für die Durchsetzung der Normen teilen. Die Flaggenstaaten haben die Pflicht, den Schiffbau und die Ausrüstung zu überwachen. Hier sind die Kontrollrechte des Küstenstaates auf die Prüfung der Zertifikate beschränkt. Kommt der Flaggenstaat seiner Ubberwachungspflicht nicht nach, so kann das Schadenersatzansprüche ihm ge-

66 in Doc. A/Conf. 62/C. 3/L. 14 Add. 1.

67 Doc. A/AC. 138/SC. III/L. 48.

68 Doc. A/AC. $138 / \mathrm{SC}$. III/L. 46.

69 Doc. NConf. 62/C. 3/L. 7. 
genüber nach sich ziehen. Insofern soll durch die Verschärfung der Pflichten der Flaggenstaaten die Durchsetzung der Umweltschutzvorschriften erzwungen werden. Das Hauptgewicht der küstenstaatlichen Befugnisse liegt dagegen bei der Úberwachung seiner Küstengewässer. So kann er gegen Schiffe vorgehen, die gegen die Umweltschutzvorschriften im Bereich der Küstengewässer verstoßen haben, und Schiffen, die keine Sicherheitszertifikate besitzen, den Zugang zu Küstengewässern und Häfen verwehren. Gleichzeitig werden die Untersuchungsbefugnisse des Küstenstaates auf der Hohen See erweitert.

\section{Zusammenfassung}

Bei einer Würdigung der Staatenpraxis sowie der Vorschläge, soweit sie im Meeresbodenausschuß oder auf der Seerechtskonferenz vorgelegt wurden, sind die gesamten geplanten Veränderungen im Seerecht mit in Rechnung zu stellen. Sollte die Seerechtskonferenz beispielsweise eine Ausdehnung der Küstengewässer bis auf $200 \mathrm{sm}$ vorschlagen, so würde dies auf jeden Fall das Problem der nationalen Umweltschutzkompetenzen auf Hoher See relativieren, da Kompetenzen jenseits der 200-sm-Begrenzung nicht beansprucht werden. Es stünde dann den Küstenstaaten offen, Regelungen zum Schutze der maritimen Umwelt im Bereich der neugeschaffenen Küstengewässer zu erlassen. Die einzige Beschränkung läge nach dem bisher geltenden Konzept der Küstengewässer für die Küstenstaaten darin, daß derartige Vorschriften wirklich dem Umweltschutz dienen und nicht diskriminierend wirken dürften. Dies ergibt sich aus dem normativen Zusammenspiel von Art. 17 des Übereinkommens über die Küstengewässer und die Anschlußzone und von Art. 24 des Übereinkommens über die Hohe See. Dennoch würde eine derartige Lösung ohne $Z$ weifel für die Schiffahrt außerordentlich weitreichende Einschränkungen mit sich bringen, da damit rund ein Drittel der gesamten Meeresfläche und vor allem alle Meerengen der nationalen Kontrolle unterstellt würden. Selbst wenn die Seerechtskonferenz dem Vorschlag Maltas folgte, und Umweltschutzregelungen nur im Rahmen vernünftiger Grenzen und unter Beachtung bestehender völkerrechtlicher Normen - einschließlich der Kontrollmöglichkeiten durch ein Meeresgericht - zuließe, wäre eine starke nationale Ausprägung der einzelnen maritimen Umweltschutzregelung nicht zu verhindern. Jede derartige mehr oder minder eigenständige nationale Regelung auf dem Gebiet des maritimen Umweltschutzes birgt, zumal wenn sie Konstruktion, Ausrüstung, Bemannung der Schiffe betrifft oder Regelungen für die Ladung enthält, die Gefahr einer substantiellen Zersplitterung der auf die Schiffahrt bezogenen Normen und bedeutet damit für diese ein Hemmnis. Die Seeschiffahrt ist mit Rücksicht auf ihre großräumigen Operationen darauf angewiesen, zumindest hinsichtlich der genannten Punkte einen möglichst weltweit homogenen Rechtszustand vorzufinden, da es ihr unmöglich ist, diese Merkmale differierenden oder sogar sich widersprechenden regionalen Forderungen während der Fahrt anzupassen. Insofern besteht die Befürchtung, daß mit Hilfe der Ausdehnung der Küstengewässer auf $200 \mathrm{sm}$ Sonderbezirke geschaffen werden, die der allgemeinen Schiffahrt praktisch verschlossen bleiben. Damit würde aber die Bedeutung der See als wesentliches derzeit noch intaktes mondiales Kommunikationselement eingeschränkt, wenn nicht sogar aufgegeben. 
Wesentlich weniger einschneidend als die bisher behandelten präventiven Maßnahmen wirken demgegenüber unterschiedliche nationale Verbote (z. B. unterschiedlich strenges Verbot, Oll abzulassen), da sich die Schiffahrt darauf eher einrichten kann. Eine Teilung der Regelungskompetenzen dergestalt, daß die Küstenstaaten entsprechende Verbote erlassen, während die Vorschriften für Bau, Ausrüstung und Bemannung international erfolgen, erscheint aber nicht realisierbar. Denn auf lange Sicht wird sich die Verschmutzung der See von Schiffen aus nur durch die Einführung der letztgenannten präventiven Maßnahmen verwirklichen lassen.

Die gleichen Bedenken wie gegen die Errichtung von Küstengewässern bis zu $200 \mathrm{sm}$ Breite bestehen gegenüber der Anerkennung von Umweltschutzzonen nach dem Vorbild Kanadas, denn praktisch beansprucht der Küstenstaat hier, zumindest was den Umweltschutz betrifft, die gleichen Rechte wie ein Küstenstaat in seinen Hoheitsgewässern. Im Grunde genommen handelt es sich bei derartigen Umweltschutzzonen lediglich um eine Vorstufe, die sich nach und nach in ein echtes Küstengewässer verwandeln dürfte. Hinzu kommt, daß vor allem der Vorschlag Kanadas nur eine sehr lockere Bindung an völkerrechtliche Umweltschutznormen vorsieht. Von ihnen soll schon bei ökologischen oder geographischen Besonderheiten der Region abgewichen werden können. Diese Generalklausel ist so weit, daß sie praktisch für alle küstennahen Gebiete die Schaffung von Sonderregelungen erlaubt; dies gilt insbesondere für Zonen mit starkem Schiffsverkehr, da dieser durchaus die Gefahr einer besonderen ökologischen Belastung mit sich bringt. Es wäre daher für die Küstenstaaten mit Hilfe dieses Konzepts ohne weiteres möglich, für derartige Regionen, aber auch für alle Meerengen besonders strenge Anforderungen hinsichtlich Konstruktion, Bemannung und navigatorischer Ausrüstung der Schiffe einzuführen.

Im übrigen ist zu bezweifeln, ob die Errichtung von Umweltschutzzonen oder die Erweiterung der Küstengewässer dem Interesse eines effektiven maritimen Umweltschutzes gerecht würde. Es ist schon fraglich, ob viele Staaten über die technischen und finanziellen Voraussetzungen verfügen, um derartige großräumige Gebiete zu überwachen und bei Ölunfällen wirksame Gegenmaßnahmen zu ergreifen $^{70}$. Ein Beispiel dafür bieten die Ausführungen des Vertreters von Chile auf der Seerechtskonferenz zum Metula-Zwischenfall in der Magellan-Straße. Obwohl Chile sonst für eine Stärkung küstenstaatlicher Kompetenzen plädiert, verlangt es nunmehr nach dem Eindruck dieses Tankerunglücks und den daraus entstandenen Folgen eine stärkere internationale Zusammenarbeit.

Gleichen Bedenken begegnen naturgemäß die Vorschläge, die den Küstenstaaten präventive Regelungskompetenzen hinsichtlich der Schiffahrt im Rahmen des Wirtschaftskonzepts übertragen wollen. Die bisher eingebrachten Vorschläge sehen allerdings in der Regel eine geringfügig stärkere Bindung an international entwikkelte Regelbestimmungen vor, als die Verfechter des Umweltschutzzonenkonzepts. Dennoch bleibt auch bei diesen Vorschlägen fraglich, ob mit Hilfe solcher Bindungen eine Zersplitterung des maritimen Umweltrechtes effektiv verhindert werden kann.

Soweit dagegen in den Wirtschaftszonen eine Regelung des Meeresbergbaus im Interesse der maritimen Umwelt erfolgt, bedeutet dies keine zusätzliche Einschrän- 
kung für den Bergbau. Insofern ist die Situation anders als bei der Schiffahrt. Bei den Anlagen des Meeresbergbaus handelt es sich bislang in aller Regel um stationäre Anlagen oder Einrichtungen mit geringer Mobilität. Sie können sich daher eher als Schiffe auf nationale Gesetzgebung einstellen. Kurz gesagt, sie benötigen nicht die See als Kommunikationselement, sondern als Plattform. Das einzige Bedenken gegen eine völlige Übertragung derartiger Regelungskompetenzen an die Küstenstaaten liegt darin, daß diese unter Umständen ihre Anforderungen tief ansetzen, zumal, wenn sie den Aufbau einer heimischen Industrie fördern und nicht mit Investitionen für den Umweltschutz belasten wollen. Dieser Gesichtspunkt wird sogar in einigen Staatenvorschlägen, vor allem aus den Reihen der Entwicklungsländer, ausdrücklich angesprochen. Die Berücksichtigung derartiger - vielleicht auch verständlicher - Interessen würde ohne $Z$ weifel $\mathrm{zu}$ einer Wettbewerbsverzerrung zwischen den Staaten führen und die Effektivität eines weltweiten Umweltschutzes beeinträchtigen. Auf der anderen Seite wären aber Konzessionen in diesem Bereich weit weniger gravierend, als wenn auch bei den an die Schifffahrt gerichteten Anforderungen auf entsprechende Interessen der Entwicklungsländer Rücksicht genommen würde. Denn anders als bei der mobilen Verschmutzungsquelle des Schiffes, treffen die Auswirkungen des Meeresbergbaus in erster Linie den Küstenstaat selbst, auch wenn Einflüsse auf Nachbarstaaten oder die Hohe See zu erwarten sind. Um wenigstens den beiden letztgenannten Gefahren ansatzweise $z u$ begegnen, wäre es daher erforderlich, einen nicht zu hoch angesetzten internationalen Mindeststandard zu schaffen, dem die Anlagen des Meeresbergbaus genügen müssen. Im übrigen ist es aber durchaus erwägenswert, auf die sozialen und wirtschaftlichen Unterschiede der einzelnen Länder Rücksicht zu nehmen und ihnen zu überlassen, welche Anforderungen sie an den Meeresbergbau in der Wirtschaftszone im Interesse des Umweltschutzes stellen wollen. Entsprechende Konzessionen würden sicherlich eine Einigung erleichtern helfen.

Aus den bisherigen Ausführungen wird deutlich, daß Schiffahrt und Meeresbergbau unterschiedliche Vorkehrungen zum Schutz der maritimen Umwelt erfordern. Während die Regelungskompetenzen bei dem Meeresbergbau durchaus, wie es viele Staaten fordern, in die Hände der Küstenstaaten gelegt werden könnten, würde das gleiche Konzept bei der Schiffahrt gefährliche Folgen haben. Dieser Úberlegung trägt der bereits erwähnte Vorschlag der USA ${ }^{71}$ Rechnung. Sein Konzept hat den Vorteil, daß es die Einheitlichkeit der völkerrechtlichen Umweltschutzregelung hinsichtlich der Schiffahrt wahrt und ihr damit keine besonderen Einschränkungen durch die Errichtung von Sonderregionen auferlegt. Gleichzeitig wird die ausschließliche Durchsetzungskompetenz der Flaggenstaaten für die Umweltschutzgebiete außerhalb der 12-sm-Küstengewässer aufgegeben, indem nunmehr auch die sog. Hafenstaaten Vergehen in diesem Bereich verfolgen sollen. Insofern dehnt sich praktisch die Polizeibefugnis der Hafenstaaten auch auf die Hohe See aus. So erfolgversprechend dieses System erscheinen mag, es begegnet doch gewissen Bedenken. Im Grunde genommen wird damit das Konzept aufgegeben, daß auf Hoher See die Schiffe nur der Jurisdiktion ihres Flaggenstaates unterliegen. Verstöße gegen den Umweltschutz werden insofern praktisch mit Piraterie oder Sklavenhandel auf gleiche Stufe gestellt. Ob dies wirklich gerechtfertigt ist, erscheint zumindest mit Rücksicht auf den verhältnismäßig geringen Anteil, den die

71 Doc. A/AC. 138/SC. III/L. 40. 
Schiffahrt an der maritimen Umweltverschmutzung hat, fraglich. Man sollte berücksichtigen, daß die Ausübung nationaler Kompetenzen hinsichtlich von Vergehen fremder Schiffe auf Hoher See auch in der Form des Hafenstaatskonzeptes einen Eingriff in den Grundsatz der Meeresfreiheit enthält. Denn das Flaggenstaatsprinzip ist als ein Ausfluß dieses Grundsatzes anzusehen ${ }^{72}$. Außerdem bedeutet die Anerkennung von Untersuchungs- und Strafkompetenzen des Hafenstaates ohne Zweifel einen Eingriff in die Souveränität der Flaggenstaaten ${ }^{73}$. Mit Rücksicht darauf, daß die meisten Staaten ängstlich darauf bedacht sind, ihre Souveränität zu wahren, ist es sehr fraglich, ob sich ein Vorschlag nach dem Konzept der USA, so praktikabel und wirksam er auch ist, durchsetzen kann. Will man derartige Bedenken vermeiden, so erscheint es nur sinnvoll, die Verantwortlichkeit der Flaggenstaaten zu verschärfen, um sie auf diese Weise anzuhalten, ihren Pflichten gewissenhaft nachzukommen und die Schiffahrt stärker zu überwachen. Dafür bietet der Vorschlag der BRD ${ }^{74}$ einen Ansatz.

Nach diesem Vorschlag sind die Flaggenstaaten gehalten, dafür zu sorgen, daß die Verpflichtungen aus dem Umweltschutzrecht von Schiffen ihrer Flagge befolgt werden. Die Staaten machen sich gegenüber anderen Staaten und deren Staatsangehörigen schadensersatzpflichtig, wenn sie ihrer Prüfungspflicht nicht genügen. Kontrollpflichten enthält bereits das IMCO-Übereinkommen von 1972, dessen Gedanken der Vorschlag der BRD aufgreift und weiter verfolgt. Die Verpflichtung zum Schadensersatz wegen Verletzung der Aufsichts- bzw. Prüfungspflichten ist dagegen in dieser Form relativ neu ${ }^{75}$. Sie läßt sich damit begründen, daß der betreffende Staat seine ihm gegenüber der Staatengemeinschaft insgesamt oder gegenüber einzelnen Staaten obliegende Pflicht, Umweltschäden aus dem Bereich seiner Jurisdiktion zu vermeiden, nicht erfüllt hat ${ }^{76}$. Das Bestehen einer derartigen völkerrechtlichen Pflicht kann allerdings bislang noch nicht als gewohnheitsrechtlich gesichert angesehen werden ${ }^{77}$. Insofern ist es richtig, daß sie nach dem Vorschlag der BRD durch Vertrag begründet werden soll ${ }^{78}$. Es handelt sich bei dem von der BRD vorgeschlagenen Konzept übrigens nicht um den Fall einer Gefährdungshaftung, so daß der Staat für Umweltschäden von Schiffen unter seiner Flagge ohne weiteres einstehen müßte. Sondern es soll lediglich eine Schadensersatzpflicht begründet werden, die daraus resultiert, daß der Flaggenstaat seinen Prüfungspflichten nicht genügt hat und dies kausal für eine Schädigung der Umwelt

\footnotetext{
72 Riphagen, The Jurisdiction of the Coastal State, in: The Future of the Law of the Sea, edited by Bouchez and Kaijen, The Hague 1973, S. 154 (156).

73 du Pontavice, Pollution, in: The Future of the Law of the Sea, edited by Bouchez and Kaijen, The Hague 1973, S. 104 (135).

74 Doc. A/Conf. 62/C. 3/L. 7 .

75 Eine weitergehende Schadensersatzpflicht des Staates sieht beispielsweise der Vorschlag der UdSSR vor (Doc. A/AC. 138/SC. III/L. 32).

76 Caflisch, a. a. O., S. 216.

77 Vgl. dazu die Trail Smelter Arbitration vom 11. 3. 1941, United Nations Report of International Arbitral Awards, Vol. III, S.1905. Caflisch a. a. O., S. 215, ist allerdings der Meinung, auch dieser Schiedsspruch enthalte keinen. Beleg für das Bestehen einer völkerrechtlichen Pflicht, schädliche Umwelteinflüsse, die auf fremde Territorien übergreifen, zu vermeiden, a. A. Schultheiss a. a. O., S. 45. Goldie, International Principles of Responsibility for Pollution, in: Columbia Journal of Transnational Law, Vol. 9 (1970), S. 283 (306).

Auch Bothe, Umweltschutz als Aufgabe der Rechtswissenschaft, in: ZaöRV 1972, S. 483 (508) vertritt die Ansicht, daß eine entsprechende Pflicht erst entwickelt werden muß.

78 Ebenso, wenn auch nicht ausdrücklich auf die Schiffahrt bezogen, die Vorschläge von Malta (Doc. A/AC. 138/SC. III $/$ L. 33), Kenia (Doc. A/AC. 138/SC. III/L. 41), UdSSR (Doc. A/AC. 138/SC. III/L. 32).

$\mathrm{Zu}$ unterscheiden von der angesprochenen Staatenverpflichtung ist die zivilrechtliche Haftung (Gefährdungshaftung) der Schiffseigner (International Convention on Civil Liability for Oil Pollution Damage (ILM Vol. 9 (1970), S. 45). Verschiedene Konventionen treffen eine ähnliche Regelung für radioaktive Schäden auf See (International Convention on the Liability of Operators of Nuclear Ships, 1962; Convention Relating to Civil Liability in the Field of Maritime Carriage of Nuclear Material, 1963 (BT - Drucksache 7/2182, S. 26; 75).
} 
geworden ist. Allerdings muß nicht der Geschädigte die Kausalität zwischen Nichterfüllung der Prüfungspflicht und Schaden nachweisen, sondern es liegt bei dem in Anspruch genommenen Staat, das Fehlen einer derartigen Kausalität darzutun. Mit dieser Umkehrung der Beweislast begegnet der Vorschlag der BRD von vornherein einem der möglichen Bedenken gegen diesen Lösungsweg. Dennoch bleibt es fraglich, ob damit ein wirklicher Druck auf die Flaggenstaaten ausgeübt werden kann, ihre Schiffe unter Umweltschutzgesichtspunkten zu überwachen. Ein stärkeres Druckmittel würde in dieser Hinsicht ohne Zweifel die Einführung einer Gefährdungshaftung bedeuten, wie sie beispielsweise für die von Weltraumgegenständen hervorgerufenen Schäden vorgesehen ist ${ }^{79}$. Auf der anderen Seite wäre die Einführung einer Gefährdungshaftung mit der Folge, daß der Staat für jede Umweltverschmutzung durch Schiffe unter seiner Flagge einzustehen hat, nicht ganz angemessen. Denn eine solche Haftungsform ist nur dann angebracht, wenn es sich um neuartige Tätigleiten handelt, deren Auswirkungen unabsehbar sind und den Keim für bedeutende Schädigungen in sich tragen ${ }^{80}$. Vor allem erscheint die Einführung einer derartigen Haftung dann nicht sinnvoll, wenn, wie es bei Umweltverschmutzung von Schiffen in der Regel der Fall sein wird, das auslösende Moment des schädigenden Ereignisses das Fehlverhalten beteiligter Personen ist ${ }^{31}$. Es ließe sich aber beispielsweise eine Gefährdungshaftung für Ölleitungen auf dem Meeresboden begründen, ohne daß dadurch die betreffenden Staaten unbillig belastet würden ${ }^{82}$. Hinsichtlich der Schiffahrt sollte es allerdings bei dem von der BRD vorgeschlagenen Konzept der Verursachungshaftung bleiben.

Ein Problem läßt der Vorschlag der BRD jedoch offen. Er berücksichtigt nicht, daß möglicherweise weder ein anderer Staat noch fremde Staatsangehörige, sondern lediglich die Hohe See geschädigt worden ist. Aber auch in diesem Fall ist die Zahlung von Schadensersatz erforderlich, um die eingetretenen Schäden beseitigen zu können. Mit diesem Gesichtspunkt beschäftigen sich, wenn auch in allgemeiner Form, die Vorschläge von Kenia und Malta. Sie sehen zutreffend vor, daß die Interessen der Staatengemeinschaft auf der Hohen See durch eine internationale Behörde wahrgenommen werden müssen. Ob der Vorschlag der BRD allerdings insgesamt gesehen Beifall bei denjenigen Staaten finden wird, die auf eine Erweiterung ihrer küstenstaatlichen Kompetenzen auch im Umweltschutzbereich dringen, ist zweifelhaft. Denn im Grunde genommen wird durch den Vorschlag der BRD an diesem entscheidenden Punkt - sieht man einmal von der Verstärkung der küstenstaatliches Untersuchungsrechte $a b-$ nichts Wesentliches geändert. Unbefriedigend wird für diese Staaten vor allem sein, daß sie nicht selbständig gegen aktive Verstöße von Schiffen gegen die Umweltschutzvorschriften - wie z. B. das unbefugte Ablassen von $\mathrm{O} l$ - außerhalb der Küstengewässer vorgehen können ${ }^{83}$. Gleichzeitig besteht aber nach dem Vorschlag der BRD auch keine Möglichkeit, die Flaggenstaaten nachhaltig zu einer Verfolgung derartiger Vergehen anzu-

79 Vgl. dazu Art. VI, VII Weltraumvertrag i. V. mit Art. II Abkommen über die völkerrechtliche Haftung für Schäden, die durch Weltraumgegenstände verursacht werden, in: ZLW 1972, S. 163.

80 Ausführlich dazu Goldie a. a. O., S. 317

81 Dennoch sieht der Vorschlag der UdSSR (Doc. A/AC. 138/SC. III/L. 32, Art. 2, 3 eine Schadensersatzpflicht des Staates für alle die Umwelt beeinträchtigenden Tätigkeiten im Bereich seiner Hoheitsgewalt vor. Dahinter steht offenkundig die Erwägung, daß es allein in seiner Hand liegt, derartige Aktivitäten und damit auch die daraus resultierenden Folgen zu verhindern.

82 Caflisch, a. a. O., S. 217, hält unter Vorbehalt sogar die Einführung einer Staatshaftung für Großtanker für denkbar.

83 Selbst Staaten wie Japan, die im übrigen nicht sehr auf die Erweiterung der küstenstaatlichen Kompetenzen drängen, halten in diesem Bereich die Einführung von hafen- und küstenstaatlichen Befugnissen für möglich (Doc. A/Conf. 62/C. 3/SR. 14). 
halten. Sie sind lediglich verpflichtet, den anzeigenden Staat von den getroffenen Maßnahmen zu verständigen. Es ist daher zu überlegen, inwieweit sich in diesem Bereich ein stärkerer Druck auf die Flaggenstaaten ausüben läßt ${ }^{84}$. In Betracht käme hierfür ein in dem Vorschlag der USA $^{85}$ angesprochener Gedanke. Danach kann ein Küstenstaat nach Einschaltung eine Streitschlichtungsstelle gegen alle Schiffe unter der Flagge eines bestimmten Staates, der die Beachtung der internationalen Umweltschutzvorschriften bei seinen Schiffen nicht durchsetzt, Sondermaßnahmen ergreifen. Der Einbau derartiger küstenstaatlicher $Z_{\text {wangsrechte in }}$ eine zukünftige Konvention zum Schutz der maritimen Umwelt könnte unter Umständen einige Staaten dazu bewegen, von dem Zonenkonzept oder vergleichbaren Vorstellungen Abstand zu nehmen.

Von der bisher besprochenen Problematik zu unterscheiden ist die Frage, welche Rechte den Küstenstaaten zustehen sollen, wenn als Folge eines Tankerunfalls ihren Küsten schwerwiegende Gefahren drohen. Hier sollte im Prinzip das von der IMCO $^{86}$ vorgeschlagene Konzept der küstenstaatlichen Eingriffsrechte nicht verlassen werden, zumal es sich dabei nur um eine Konkretisierung des völkerrechtlich anerkannten allgemeinen Notstandsrechts handelt. Es könnte hier aber durchaus der Vorschlag der USA in Erwägung gezogen werden, das komplizierte Konsultationssystem aufzugeben, das ein rechtzeitiges und effektives Eingreifen behindert.

Auch hinsichtlich der Abfallbeseitigung auf Hoher See sollte es bei dem Verfahren der London- 87 bzw. Oslo-88 Konvention verbleiben. Die Abfalibeseitigung auf Hoher See ist am besten von jedem Staat selbständig zu kontrollieren. Insofern ist es richtig, daß die Vernichtung von Abfallstoffen im Meer von einer vorherigen Genehmigung abhängig gemacht wird.

Die Oslo-Konvention weist in dieser. Hinsicht allerdings einen Vorteil auf, als sie, zumindest ansatzweise, eine internationale Überwachung der nationalen Genehmigungen erlaubt. Ein derartiger Kontrollmechanismus sollte auch in die zukünftige Konvention über den Umweltschutz auf See aufgenommen werden, um wenigstens die Einhaltung bestimmter Mindestanforderungen durch die Staaten zu gewährleisten. Die Tendenz auf der Seerechtskonferenz deutet allerdings leider, soweit sich das bisher absehen läßt, nicht in diese Richtung. Man scheint vielmehr den Küstenstaaten größere Freiheiten zubilligen zu wollen.

Eine besondere Problematik stellt schließlich noch die Bekämpfung der maritimen Umweltverschmutzung von Land aus dar. Für sie entsprechende internationale Regelungen zu erlassen, ist wohl nicht so ohne weiteres möglich. Die meisten Staaten werden dies als einen unzulässigen Eingriff in ihre Souveränität ansehen und sich schon aus diesem Grund gegen derartige Bestrebungen wehren. Vor allem die Entwicklungsländer befürchten, dies könne ihnen den Aufbau einer eigenen Industrie erschweren. Nicht umsonst haben sie bereits auf der Umweltschutzkonferenz in Stockholm wie auch in ihren Vorschlägen auf der Seerechtskonferenz auf ihre Sonderstellung hingewiesen und eine Berücksichtigung ihrer Probleme gefordert. Dennoch läßt sich diese Frage aus dem Gesamtkomplex nicht ausklammern, wenn nicht die Regelung des maritimen Umweltschutzes zum Scheitern

\footnotetext{
84 Das würde in der Tendenz den auf der Konferenz geäußerten Vorstellungen der BRD entsprechen (Doc. A/Conf. 62/C. 3/SR. 5, S. $15 \mathrm{ff}$.)

85 Doc. A/AC. 138/SC. III/L. 40 .

86 International Convention Relating to Intervention on the High Seas in Cases of Oil Pollution Casualities, ILM, Vol. 9 (1970), S. 25.

87 ILM, Vol. 11 (1972), S. 1291.

88 ILM, Vol. 11 (1972), S. 262.
} 
verurteilt sein soll: Die Konferenz müßte doch versuchen, wenn auch vielleicht abgeschwächt durch eine Übergangsregelung, Mindeststandards festzuschreiben und das Verbot der Verschmutzung der See von Land aus in die zukünftige Konvention aufzunehmen. Die Einführung einer Schadensersatzpflicht, wie sie z. B. der Vorschlag von Malta ${ }^{89}$ enthält, dürfte dagegen zumindest jetzt noch nicht realisierbar sein.

89 Doc. A/AC. 138/SC. III/L. 33. 


\section{The Protection of the Marine Environment}

\section{BY RÜDIGER WOLFRUM}

The settlement of the protection of the marine environment at the Third Conference on the Law of the Sea will gain importance due to possible effects on shipping and sea-mining. Previous attempts to control the increasing pollution of the sea had little effect, and have been strongly criticised. Yet only a few states, for example Canada, have established pollution prevention zones adjoining their own coastal waters, thereby claiming far-reaching regulation and control competences. Canada justifies its action by stating that an effective protection of the marine environment can only be achieved by this method.

At the Third Conference on the Law of the Sea, various ideas to combat the pollution of the marine environment were put forward, based on two completely different concepts. The first group demands a drastic extension of coastal state competences, which would allow the coastal states to look after the marine environment outside their territorial waters. It is not clear whether these competences concern only permission to control, or also permission for regulations and how far they will be bound by international standards.

Such an increase of coastal state competences would cause difficulties for shipping. This applies, in particular, to national regulations dealing with the construction, equipment, manning and loading of ships. Special areas of the sea may be closed to shipping in general. Moreover, it would be difficult to accommodate this type of competence under the present principle of international Law of the Sea. This is based on the freedom of the High Seas, which provides that ships on the High Seas are under the jurisdiction of their own flag-state. The transfer of protection of the environment competences to coastal states with regard to sea-mining seems less extreme. However, it is to be feared that states in the process of industrialization will not take sufficient consideration of the demands of the protection of the environment if no internationally binding minimum standards are set.

The other position of the Conference on the Law of the Sea favours the creation of an international authority to work out the regulations for the protection of the marine environment, and to secure general international standards.

The best solution would be the inclusion into the existing system of the Law of the Sea of a regulation for the protection of the marine environment, based on the principle of the flag-state authority. This has been suggested by the Federal Republic of Germany. The obligations of the flag-states shall thereby be intensified; they become liable, if they fail to fulfill their responsibilities.

Greater consideration should be given to this idea at the Conference on the Law of the Sea. It promises an effective protection of the environment of the sea without, at the same time, limiting the concept of the freedom of the High Seas. 